\title{
Concurrent Salmonella Mycotic Abdominal Aneurysm and Empyema Thoracis: A Rare Coincidence
}

\author{
Chia-Ter Chao
}

Renal Division, Department of Internal Medicine, National Taiwan University Hospital Jin-Shan Branch, New Taipei, Taiwan, ROC

\section{Key Words}

Mycotic abdominal aortic aneurysm · Salmonella enteritidis . Empyema - Empyema thoracis - Distant suppurative foci

\begin{abstract}
Objective: To present a case of Salmonella bacteremia with a rare metastatic focus. Clinical Presentation and Intervention: A 61-year-old male presented with a suicide attempt after ingestion of half a bottle of toilet cleaner. He had a medical history of intravenous drug abuse and major depression. Persistent Salmonella enteritidis bacteremia occurred during his hospitalization, and a search for the primary source of infection disclosed a mycotic abdominal aortic aneurysm. A suppurative lesion was also noted over the left pleural space, and Salmonella empyema was confirmed after thoracentesis. He underwent video-assisted thoracoscopic surgery and endovascular repair of the abdominal aortic aneurysm and was placed on long-term antibiotics, without recurrence. Conclusion: S. enteritidis involvement of the cardiovascular system is a rare coincidence and results in significant morbidity and mortality. In addition, the phenomenon of secondary metastatic infective foci involving the pleural space has an even lower frequency than that of cardiovascular involvement. Therefore, a high index of suspicion and prompt treatment from the treating physicians are strongly recommended.

(c) 2014 S. Karger AG, Basel
\end{abstract}

\section{Introduction}

Salmonella, a member of the family Enterobacteriaceae, is a motile, non-spore-forming, Gram-negative bacteria with a high incidence of localized suppurative infections [1]. Gastroenteritis is the most common presentation of Salmonella infection (60-70\%), but extraintestinal manifestations usually occur after bacteremia [2]. Pleuropulmonary involvement accounts for only $15 \%$ of focal manifestations during Salmonella infections, among which pulmonary parenchymal involvement is predominant, while empyema accounts for a minority of cases [3]. Herein, a rare case of Salmonella bacteremia with a concomitant mycotic aneurysm and thoracic empyema is presented.

\section{Case Report}

A 61-year-old male presented for evaluation of a possible esophageal corrosive injury after ingestion of half a bottle of toilet cleaner. He had a medical history of intravenous drug abuse and major depression. Esophagogastroduodenoscopy and upper gastrointestinal radiography did not reveal esophageal perforation or leakage. A psychiatric consultation was obtained, and the antidepressive regimen was adjusted. However, persistent fever was noted during admission, and two sets of blood cultures grew Salmonella enteritidis. The patient had mild diarrhea but was negative for human immunodeficiency virus. An initial chest X-ray revealed mild haziness

\begin{tabular}{ll}
\hline KARGER & $\begin{array}{l}\text { ( ) 2014 S. Karger AG, Basel } \\
1011-7571 / 14 / 0235-0482 \$ 39.50 / 0 \quad \text { Karger }\end{array}$ \\
$\begin{array}{l}\text { E-Mail karger@karger.com } \\
\text { www.karger.com/mpp }\end{array}$ & $\begin{array}{l}\text { Thisis an Open Access article licensed under the terms of the } \\
\text { Creative Commons Attribution-NonCommercial 3.0 Un- } \\
\text { ported license (CC BY-NC) (www.karger.com/OA-license), } \\
\text { applicable to the online version of the article only. Distribu- } \\
\text { tion permitted for non-commercial purposes only. }\end{array}$
\end{tabular}

Chia-Ter Chao

Renal Division, Department of Internal Medicine

National Taiwan University Hospital Jin-Shan Branch

No. 51, Nan-Shih, Jin-Shan District, New Taipei 20844, Taiwan (ROC)

E-Mail b88401084@gmail.com 


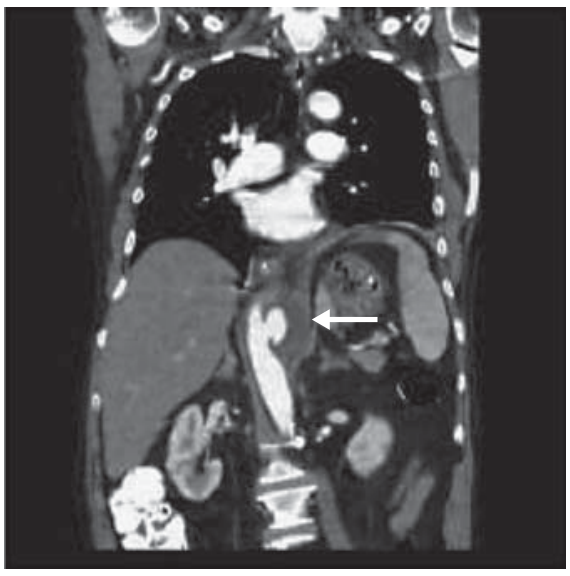

Fig. 1. Coronal slice of an abdominal CT with contrast demonstrating a 'pseudokidney' sign (arrow) with an aneurysmal sac and pseudolumen as the renal contour. The true lumen appears as the renal hilum. The hypodense area within the pseudokidney represents the mural thrombus in the aneurysm.

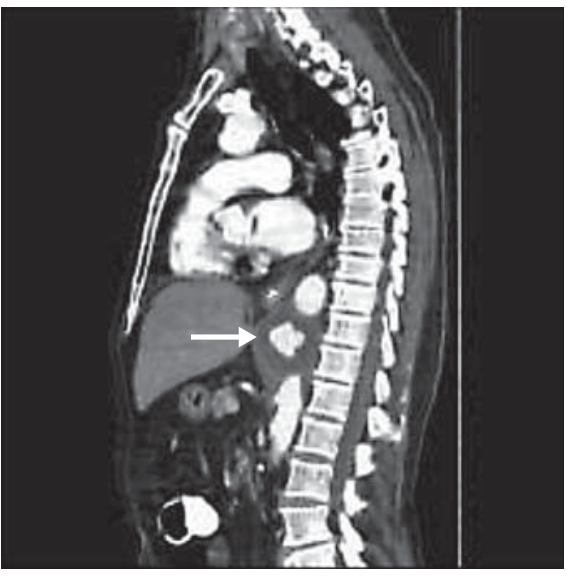

Fig. 2. Sagittal slice of an abdominal CT with contrast demonstrating the abdominal aortic aneurysm as a 'target' sign (arrow) and the hypodense area surrounding the contrast-filled true lumen as a thrombus.

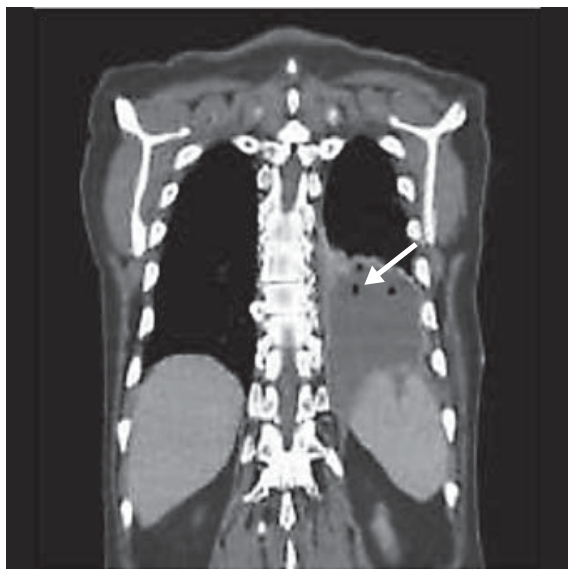

Fig. 3. The arrow shows an air bubble in the left pleural space suggestive of suppurative (gas-forming) foci with pleural effusion of heterogeneous density indicating empyema. over the left lower lung field. A thoracoabdominal CT with contrast disclosed a 6-cm saccular abdominal aortic aneurysm (fig. 1, 2). A moderate left pleural effusion was also discovered. Thoracentesis was performed, and the effusion culture also grew S. enteritidis (fig. 3 ). The patient refused to undergo aortic bypass surgery, and subsequently we arranged endovascular aneurysm repair of the mycotic aneurysm. His empyema was managed with video-assisted thoracoscopic surgery. He completed a prolonged ciprofloxacin therapy of 6 weeks after an operation for eradication of the Salmonella infection. There was no recurrence within the following 6 months as confirmed by negative blood cultures.

\section{Discussion}

This was a case of a rare coincidence of concomitant Salmonella mycotic aneurysm and thoracic empyema, resulting from Salmonella bacteremia owing to gastrointestinal mucosal bacterial translocation. It was necessary to search for additional metastatic foci other than the common infestation sites during Salmonella infection.

Radiologic differential diagnosis of aortic aneurysm is a rather underrecognized issue. Mycotic abdominal aortic aneurysms often present in saccular forms with a multilobulated appearance and tend to interrupt arterial wall calcification on CT as in this case. On the contrary, atherothrombotic aneurysms are frequently fusiform, coexist with atherosclerotic wall calcification, and assume a more regular internal contour [4]. In our case, the aortic aneurysm was saccular and contained focal fluid accumulation, thereby corresponding with a mycotic origin.

Patients with nontyphoidal Salmonella infections frequently present with gastroenteritis, bacteremia, arthritis, bony infections, and most importantly cardiovascular infections [5]. Cardiovascular infections reportedly occur in $25 \%$ of patients with Salmonella bacteremia, and patients with atherosclerosis, especially atherosclerotic aortic aneurysms, are most susceptible to Salmonella inhabitation [5]. Our patient had widespread atherosclerosis (detected by calcification of vascular walls over multiple major vessels) and presented with unremitting fever, a characteristic feature of Salmonella vascular infections [5, 6]. The mortality incidence can be as high as $45 \%$ [6].

On the contrary, S. enteritidis empyema is a rare occurrence, constituting less than $1 \%$ of all cases of Salmonella infection [7]. Case reports in the literature suggest that factors such as advanced age, pleuropulmonary neoplasms, immunosuppression, and preexisting pleural pathology can predispose to pleuropulmonary involvement of salmonellosis, among which $S$. enteritidis empyema occurs in less than $10 \%$ of cases $[3,7]$. Most serotypes isolated from empyema include S. typhimurium, S. choleraesuis, and S. paratyphi, but isolation of $S$. enteritidis is quite rare [8]. Pathogenic mechanisms proposed to explain the development of S. enteritidis empyema include the spread of pul- 
monary parenchymal infections, seeding from nearby endovascular metastatic sites, or hematogenous spread during bacteremia $[3,7,9,10]$. The suspected port of infection in the reported case might have been via a hematogenous route due to gastrointestinal translocations caused by the esophageal corrosive injury of the patient, resulting from the ingestion of toilet cleaner, which is reportedly very rare, with an overall mortality of $63 \%$ [3]. In this case, it became much easier to detect the vascular involvement because we had already identified the S. enteritidis infection, but a rarer site of dissemination, such as empyema, could be missed. Earlier identification might facilitate therapeutic planning and potentially improve the patient's prognosis.

\section{Conclusion}

This case showed S. enteritidis involvement of the cardiovascular system, a rare coincidence that results in significant morbidity and mortality. In addition, the phenomenon of secondary metastatic infective foci involving the pleural space has an even lower frequency compared to that of cardiovascular involvement. Therefore, a high index of suspicion and prompt treatment from the treating physicians are strongly recommended.

\section{References}

$>1$ Crum NF: Non-typhi Salmonella empyema: case report and review of the literature. Scand J Infect Dis 2005;37:852-857.

$\checkmark 2$ Al-Obeid K, Al Khalifan NN, Jamal W, et al: Epididymo-orchitis and testicular abscess caused by Salmonella enteritidis in immunocompromised patients in Kuwait. Med Princ Pract 2006; 15:305-308.

3 Aguado JM, Obeso G, Cabanillas JJ, et al: Pleuropulmonary infections due to nontyphoid strains of Salmonella. Arch Intern Med 1990;150:54-56.

4 Macedo TA, Stanson AW, Oderich GS, et al: Infected aortic aneurysms: imaging findings. Radiology 2004;231:250-257.
Hohmann EL: Nontyphoid salmonellosis. Clin Infect Dis 2001;32:263-269.

-6 Fernandez Guerrero ML, Aguado JM, Arribas A, et al: The spectrum of cardiovascular infections due to Salmonella enterica: a review of clinical features and factors determining outcome. Medicine (Baltimore) 2004;83:123138.

7 de Lope ML, Batalha P, Sosa M, et al: Pleural empyema due to Salmonella enteritidis in a non-immunocompromised patient. Eur J Clin Microbiol Infect Dis 2004;23:792-793.

8 Fortún J, Domínguez A, Villarrubias J, et al: Empyema caused by Salmonella non-typhi (in Spanish). Enferm Infecc Microbiol Clin 1989;7:229-230.
9 Yang CC, Chuang FR, Wu CH, et al: Refractory Salmonella enterica serotype choleraesuis-related renal cyst infection in a patient with autosomal dominant polycystic kidney disease undergoing hemodialysis treated successfully with intracystic ciprofloxacin infusion. Med Princ Pract 2012;21:576-578.

10 Chao CT, Wu VC, Lai CF, et al: Advanced age affects the outcome-predictive power of RIFLE classification in geriatric patients with acute kidney injury. Kidney Int 2012;82:920927 